Service social

\title{
Le partenariat entre l'État et les organismes communautaires : un défi pour la formation en travail social
}

\section{Jean Panet-Raymond}

Volume 40, numéro 2, 1991

Formation et évolution de la pratique en travail social

URI : https://id.erudit.org/iderudit/706527ar

DOI : https://doi.org/10.7202/706527ar

Aller au sommaire du numéro

Éditeur(s)

École de service social de l'Université Laval

ISSN

1708-1734 (numérique)

Découvrir la revue

Citer cet article

Panet-Raymond, J. (1991). Le partenariat entre l'État et les organismes communautaires : un défi pour la formation en travail social. Service social, 40(2), 54-76. https://doi.org/10.7202/706527ar
Résumé de l'article

Ce texte présente un aperçu de l'évolution du discours sur le partenariat, issu tant des politiques gouvernementales (provinciales) que des regroupements communautaires.

Il définit ensuite les enjeux de ce partenariat entre organismes publics et communautaires avant de décrire les pratiques vécues dans les champs de la santé mentale et du maintien à domicile des personnes âgées. Le texte se termine sur quelques pistes de changement dans la formation en travail social pour que celle-ci soit en mesure de mieux préparer au travail dans un contexte de partenariat entre l'État et les organismes communautaires. 
Jean Panet-Raymond, professeur à l'École de service social de I'Université de Montréal.

\section{Le partenariat entre l'État et les organismes communautaires : un défi pour la formation en travail social}

Jean Panet-Raymond

\section{Introduction}

La crise de l'État-providence a marqué les années 80 . Les années 90 semblent être caractérisées par le partenariat de l'État avec les organismes communautaires. C'est le fruit d'un long processus amorcé dès la fin des années 70 par le gouvernement péquiste qui reprenait les modèles scandinaves de concertation état-patronat-syndicat : ce furent les fameux sommets auxquels on a finalement accepté d'inviter quelques représentants du milieu communautaire (regroupements les plus verbaux !)

Où en sommes-nous aujourd'hui ? Le discours a évolué énormément du côté des gouvernements et les discours sont restés relativement stables du côté des organismes communautaires, bien qu'il $n^{\prime} y$ ait pas de position monolithique. Quant aux pratiques mêmes de collaboration, il y en a eu une variété qui doivent être analysées de près avant de mériter l'étiquette de partenariat. L'objet de ce texte est de montrer d'abord l'évolution du discours public et communautaire sur le partenariat entre État et organismes communautaires. Ensuite, la description des pratiques dans certains champs d'activité sera faite pour en définir les enjeux et les conditions de réalisation. Enfin, la dernière partie du texte tentera de cerner les conséquences et les changements possibles pour la formation en travail social.

Mais avant d'amorcer l'analyse du discours, il faut éliminer la confusion et l'ambiguïté qui entourent le terme même de partenariat, 
qui, comme les termes concertation et communautaire, est très galvaudé.

\section{Le discours : évolution du mythe}

\section{Le discours de l'État}

La crise de l'État-providence et l'avènement du néo-conservatisme ont provoqué la production de plusieurs politiques, émanant du ministère de la Santé et des Services sociaux, sur la fin du monopole de l'État et le besoin de tenir compte des " aidants naturels ", du bénévolat et des organismes communautaires. On a fait I'apologie des réseaux naturels et des forces vives des milieux qui devaient prendre la relève de l'État endetté. La Commission Rochon a provoqué la production de plusieurs études tant par les ministères (J. Tremblay, 1987; H. Tremblay, 1987) que par des chercheurs (Lesemann et Lamoureux, 1988; Godbout et Collin, 1988; Shragge et Ladouceur, 1988). Ces études confirment les forces et les acquis pertinents des organismes communautaires, mais aussi leur fragilité et leur sous-financement chronique.

Ces études, doublées de l'extraordinaire présence des organismes communautaires aux audiences publiques de la Commission, ont certainement influencé l'énoncé du rapport final (Rochon, 1988) qui fait un portrait encore sommaire de la diversité du mouvement communautaire, mais qui reconnaît au moins sa légitimité, sa compétence et le besoin de l'associer aux politiques gouvernementales, justifiant ainsi son financement public. Ironiquement ce n'est pas dans ce rapport que l'on voit pour la première fois la reconnaissance de ce partenaire de l'État.

C'est dans le rapport Harnois (1987) sur la santé mentale que le discours sur le partenariat est le plus avancé et le plus enthousiaste. C'est la consécration et l'apologie des organismes communautaires que l'on invite à un " partenariat élargi ». Les cyniques diraient que le rapport Brunet (1987), publié quelques mois plus tôt, venait déjà préparer le terrain en limitant le rôle de l'action communautaire dans les centres locaux de services communautaires (CLSC) au soutien et à la création des organismes communautaires inscrits dans la mission et les champs d'activité prioritaires des CLSC.

Les deux énoncés de politique de la ministre Lavoie-Roux en 1989 (Politique de santé mentale; Orientations. Pour améliorer la santé et le bien-être au Québec) vont faire avancer encore un peu la définition des organismes communautaires et de la collaboration entre ceux-ci et l'État. On reconnaît leur compétence et leur complémentarité au réseau public. Mais on maintient le rôle de maître d'œuvre au ministère 
de la Santé et des Services sociaux (MSSS), qui déterminera les priorités et la planification des services à offrir. Donc, le partenariat s'inscrit dans une politique qui reconnaît l'existence d'organismes de services, d'entraide et même de défense de droits œuvrant dans le champ de la santé et des services sociaux. Le partenariat prône une participation des organismes communautaires, notamment dans la planification même de l'organisation des services : en santé mentale, ce sera la participation aux comités tripartites, remplaçant les commissions administratives des CRSSS, pour la conception des plans régionaux d'organisation de services (PROS). La notion de partenariat « équitable » du rapport Rochon a évolué pour faire état d'un financement plus stable et plus généreux mais sans avancer de chiffres précis.

Le dernier énoncé de politique est celui du ministre Côté (1990). II précise un peu plus les définitions des organismes communautaires et les critères de financement dans le projet de loi 120 discuté en 1991 (articles 254 et 257). La reconnaissance à part entière des organismes communautaires est maintenue au même titre que les établissements privés. On avance même que les programmes de perfectionnement du personnel s'adresseront aussi à celui des organismes communautaires. De plus, la réforme Côté vise à établir des modes d'évaluation de l'ensemble des établissements y compris les organismes communautaires. Et pour la première fois dans ces énoncés de politique, on avance des montants précis : augmentation de 50 millions de dollars à 90 millions sur une période de cinq ans. On consacre aussi la place des représentants d'organismes communautaires aux instances électives des régies régionales.

Voilà donc brièvement le discours public officiel sur le nécessaire partenariat avec les organismes communautaires. Il s'agit d'une politique globale voulant ouvrir le monopole institutionnel aux nouveaux partenaires. La collaboration se fait donc avant tout par un financement par l'entremise des régies sur le plan régional et local et du Ministère pour les organismes provinciaux.

Que dit-on des collaborations plus directes entre établissements publics et communautaires ? Les énoncés souhaitent évidemment les collaborations multiformes entre diverses catégories d'établissements de services et de formation, voire entre ministères. Mais le projet de loi vient limiter ces ententes en interdisant toute considération financière (article 84). Cette limite soulève donc encore la définition même du partenariat. Est-il national, régional ou local ? Est-il fondé sur une reconnaissance légale ou sur la discrétion d'un établissement local ? II semble qu'il puisse exister à des paliers différents et dans des formes différentes.

En somme, le discours étatique fait état d'un partenariat dans la planification et la réalisation des plans d'organisation de service sur 
le plan régional et local. II vise à intégrer clairement les organismes dans son plan d'ensemble, comme il vise à développer une concertation générale entre plusieurs catégories d'établissements du réseau et même d'autres champs d'activité (intersectorielle). On ne peut plus penser qu'il s'agit simplement d'une opération de cosmétique (J. Tremblay 1987 : 5) pour jeter de la poudre aux yeux des organismes encore sceptiques à l'égard des intentions du Ministère. Est-ce suffisant et est-ce acceptable pour les organismes visés?

\section{Le discours des organismes communautaires}

Selon leurs origines et leur champ de pratique, les organismes communautaires ont un discours varié (Lesemann et Lamoureux, 1988 : 184-185). Certains organismes offrant des services qui s'insèrent plus clairement dans la mission du Ministère, comme les centres d'action bénévole et certains centres communautaires pour personnes âgées, se voient assez bien insérés dans la philosophie du partenariat (Panet-Raymond et Vandal, 1990). Ils se voient tout à fait en continuité avec les services offerts notamment par les CLSC (maintien à domicile), les centres de jour et même les centres d'accueil. Mais ils demeurent inquiets de l'augmentation du fardeau qui leur revient. Des organismes comme le Forum des citoyens âgés de Montréal et l'Association québécoise pour la défense des droits des retraités et préretraités (AQDR) sont plus critiques des pratiques observées et dénoncent la tendance des organismes du réseau à utiliser les organismes communautaires comme déversoirs de leur clientèle grandissante.

Le discours d'autres regroupements comme le Regroupement des organismes communautaires jeunesse (ROCJ), I'R des centres de femmes, le Regroupement des ressources alternatives en santé mentale du Québec (RRASMQ, 1991) et la Coalition des organismes communautaires du Québec (COCQ, 1988; Parazelli, 1988) est plus critique. Issus d'une tradition plus militante de défense de droits et de promotion d'intérêts, ces regroupements acceptent la philosophie du partenariat, mais à des conditions qu'ils jugent encore peu présentes dans le discours de l'État et surtout dans les pratiques. On exige une collaboration qui reconnaisse et respecte, non pas seulement certains services complémentaires à ceux du réseau, mais aussi l'autonomie des organismes communautaires. Cette reconnaissance totale exige évidemment un financement nettement plus élevé qui consacrerait la reconnaissance de l'ensemble des activités de ces organismes ainsi que le savoir-faire de leur personnel. On demande aussi la reconnaissance d'un mode de fonctionnement qui est démocratique et qui valorise la contribution bénévole et non "professionnalisante". 
En somme, ces regroupements et d'autres dans divers champs d'activité veulent être reconnus d'intérêt public tout en restant des partenaires autonomes. C'est plus que d'être les simples déversoirs des services du réseau. On est même prêt à accepter des conditions de financement, telles que l'évaluation des services offerts, dans la mesure où les organismes sont présents dans le développement des instruments d'évaluation. Donc, en général, et cela représente un net changement d'attitude par rapport aux années 70, les organismes communautaires acceptent le discours du partenariat, mais pas dans une optique néo-conservatrice de laisser-faire de l'État (retrait et déresponsabilisation), ni dans une optique monopolisante de l'État-providence total, centralisateur et omnipuissant.

En terminant ce regard sur les discours, il faut préciser les enjeux de cette collaboration pour chacune des parties en cause.

Pour le réseau public, on peut en relever trois principaux :

- la volonté politique d'être au service de la communauté plutôt qu'à celui de ses structures et de ses programmes;

- la capacité de répondre de manière appropriée et efficace aux besoins;

- le maintien de la crédibilité des établissements face à la population et face aux organismes communautaires.

Considérant la conjoncture politique et économique, le financement et la survie dans sa forme actuelle du système public sont mis en question et cela constitue un enjeu complémentaire.

Pour les organismes communautaires, on peut relever quatre enjeux principaux :

- le respect de leur nature même;

- la qualité de leur reconnaissance publique et populaire;

- l'ampleur et les conditions de leur financement;

- leur développement autonome comme force sociale ouverte et progressiste.

Pour la population, les enjeux se centrent sur la qualité, l'efficacité et l'accessibilité des services et sur la promotion et le respect des droits, surtout des catégories les plus démunies.

Avec ces enjeux en tête, on peut se demander si les pratiques actuelles sont porteuses d'espoir !

\section{La réalité du partenariat... !}

Un discours public si généreux ne peut qu'engendrer des déceptions face à la réalité. On doit cependant noter l'augmentation con- 
sidérable du financement des organismes par le Service de soutien aux organismes communautaires du MSSS (SOC). Le budget est passé de 1,5 million de dollars à 25 millions en dix ans, de 1977 à 1987. Le ministre Côté envisage, dans sa réforme, d'augmenter ce montant de 8 millions par année pendant cinq ans pour atteindre éventuellement 90 millions de dollars. À l'intérieur de ce budget, les crédits accordés aux organismes œuvrant dans le programme de maintien à domicile des personnes âgées et handicapées sont passés de $156000 \$$ en 1979 à près de 6 millions en 1989 (rapports annuels du MSSS). II est intéressant de noter cependant que $84 \%$ de tous les organismes financés par le SOC sont indépendants, sans aucun lien contractuel avec le réseau ( $\mathrm{H}$. Tremblay, $1987: 49)$. Mais on remarque que ces organismes œuvrant dans le programme de financement du maintien à domicile sont assez fortement dépendants du financement du MSSS, car ils n'engendrent que 0,40 $\$$ de financement autonome pour chaque dollar venant du MSSS (J. Tremblay, 1987 : 49).

Dans cette partie, seront mis en évidence certaines conclusions ou facteurs déterminants qui ressortent de la collaboration régionale et locale entre les organismes publics et communautaires dans deux champs d'activité : la santé mentale (RRASMQ, 1990, 1991) et le maintien à domicile. Mais avant de regarder ces données empiriques, il n'est pas inutile de comparer les caractéristiques principales des deux catégories d'organismes.

Les éléments qui caractérisent les organismes communautaires par rapport aux organismes publics sont :

- un accent mis sur la prévention (cet élément ne se retrouve pas toujours);

- une approche globale tenant compte de l'environnement des personnes;

- une approche favorisant la prise en charge par les personnes et les groupes (entraide);

- un enracinement dans le milieu;

- une capacité de mobiliser les solidarités primaires (réseaux naturels);

- la souplesse de l'organisation;

- la capacité de s'adapter à des besoins nouveaux;

- un fonctionnement démocratique favorisant l'implication des usagers;

- une présence plus ou moins importante de bénévoles.

Ce qui caractérise les organismes publics par rapport aux organismes communautaires, ce sont les éléments suivants :

- une professionnalisation excessive laissant peu ou pas de place au bénévolat; 
- une lourdeur bureaucratique;

- I'absence d'une approche multidisciplinaire;

— un manque d'intervention à caractère préventif;

- une place restreinte laissée aux usagers;

- un fonctionnement peu démocratique.

Cette vision ( $H$. Tremblay, $1987:$ 18-22) fait ressortir l'écart entre les deux types d'organismes malgré toutes les nuances qui doivent exister sur ces éléments qui se retrouvent toujours à des degrés très variables. Ce qu'il ne faut surtout pas oublier, c'est que ces organismes ont des origines, des missions, des perspectives, des budgets, des pratiques et des cultures organisationnelles extrêmement différents.

Plusieurs s'inscrivent nettement en marge de l'intervention professionnelle. Les organismes communautaires œuvrent habituellement à des niveaux très près des préoccupations quotidiennes des clientèles avec les réseaux primaires. Cependant, plusieurs d'entre eux gardent aussi une perspective globale des problématiques en intervenant sur le plan des déterminants macro-sociaux, économiques et politiques. Cette dernière intervention se fait habituellement par l'entremise de regroupements régionaux ou nationaux.

Une collaboration entre deux types aussi divergents peut-elle être autre chose qu'un dialogue de sourds ? Est-ce qu'une telle collaboration n'est pas inévitablement vouée à l'échec malgré toute la bonne volonté des parties en présence? L'expérience révèle beaucoup d'échecs, mais aussi des réussites (Godbout et Collin, 1988) dont il faut tirer les leçons.

\section{L'exemple de la santé mentale}

Comme le rapport Harnois a suscité la création de la politique en santé mentale dès janvier 1989, c'est évidemment le champ d'action qui est le premier à tenter de vivre ce " partenariat élargi " prôné. II faut préciser que pour l'auteur du rapport ce partenariat élargi s'appliquait à plusieurs acteurs de nature totalement différente. On souhaitait associer avant tout la personne ayant besoin d'aide à sa propre solution. Déjà là il y avait un bond qualitatif important par rapport à l'approche thérapeutique traditionnelle qui était centrée sur l'intervenant professionnel. Donc, d'abord un partenariat entre intervenant professionnel et client. Ensuite, on souhaitait la participation du réseau naturel immédiat du client. Enfin, on jugeait essentiel de mettre à profit les ressources communautaires du milieu et les autres établissements publics et privés. C'est donc un vaste partenariat à dimensions multiples que l'on voulait mettre en œuvre en favorisant toujours la prise en charge dans le respect de l'autonomie de la personne et de son 
milieu. Au chapitre de l'organisation ce partenariat se traduisait par les plans d'organisation de service pour chaque région à l'intérieur desquels chaque personne aurait évidemment un plan d'intervention individuel.

Les ressources alternatives en santé mentale acceptaient le pari du partenariat dès le printemps de 1989 pour tenter de mettre en œuvre cette politique. Elles y apportaient une jeune tradition fondée sur une vision de la santé mentale, une approche, un mode de fonctionnement et des origines fort différents du réseau public. Il est important de bien saisir ces caractéristiques, car elles expliquent en partie les résultats de cette expérience de collaboration avec le réseau public.

Ce qui suit est tiré d'un bilan de cette expérience de partenariat entre établissements publics, ressources intermédiaires et ressources alternatives. Le bilan est le résultat d'une consultation d'organismes membres et non membres du Regroupement des ressources alternatives en santé mentale du Québec (RRASMQ, 1990, 1991) qui ont participé aux comités tripartites visant à la conception des plans régionaux d'organisation de services (PROS). Le bilan a été effectué par et pour le RRASMQ avec la collaboration de chercheurs universitaires. II s'agit donc d'un document qui doit alimenter le débat et la réflexion du RRASMQ afin d'en arriver à une position et de développer une stratégie d'action.

Les ressources alternatives sont issues des besoins non satisfaits du milieu : "Nous sommes issus de la communauté et impliqués avec la communauté »(RRASMQ, $1990: 7)$. Elles sont le fruit d'initiatives de parents et d'amis de personnes souffrantes et ne trouvant pas une place dans la société.

Cette origine est accompagnée d'une vision globale des personnes aux prises avec des problèmes de santé mentale :

Nous avons la conviction que le contexte économique, social et culturel dans lequel vivent les personnes constitue un des déterminants majeurs de leur état de santé physique et mentale (...). C'est pourquoi les ressources alternatives insistent sur les causes sociales, familiales, économiques, politiques et culturelles tout autant que sur les causes d'ordre biologique, physiologique, psychologique. C'est pourquoi nous refusons le découpage artificiel et arbitraire de notre approche. (RRASMQ, $1990: 6$ )

À cette vision s'ajoute une approche de l'intervention où le service n'est pas une fin en soi, où le client est condamné à n'être qu'un consommateur passif. C'est plutôt un service qui répond à un besoin certes, mais qui est également l'occasion de conscientisation. La gamme de services offerts est très variée, allant du service d'aide et d'orientation à la défense des droits, à l'hébergement, aux groupes $d^{\prime}$ entraide et à l'action communautaire. 
L'organisation communautaire doit nécessairement faire partie d'une gamme de services psycho-sociaux, faute de quoi il en sera de cette approche axée sur la personne et son milieu naturel comme il en a été de la psychiatrie communautaire : ses résultats seront minimes, un constat d'échec..." (RRASMQ, $1990: 8$ )

Enfin, les ressources alternatives favorisent un mode de fonctionnement qui poursuit cette approche, en mettant l'usager au centre de I'exercice du pouvoir démocratique dans les organismes. On accorde une place prépondérante et on privilégie des formes diversifiées de démocratie directe.

Cette approche encourage la personne en difficulté à devenir autonome, à se prendre en charge et à assumer ses responsabilités de rapports plus égalitaires avec les intervenants-es. (RRASMQ, $1990: 8$ )

En somme, le défi du partenariat avec l'État se résume ainsi :

- pour l'alternative, il s'agit de faire reconnaître son " ailleurs et autrement " de même que le financement de ses services au-delà d'une reconnaissance verbale;

- pour les personnes elles-mêmes aux prises avec des difficultés, il $s^{\prime}$ agit de vérifier la faisabilité réelle d'un partenariat égalitaire, seule garantie d'amélioration de leur condition. (RRASMQ, $1990: 9$ )

Ainsi on peut voir l'écart qui guettait cette tentative de partenariat. En pratique il s'est traduit par la création de comités tripartites sous la responsabilité des Conseils régionaux de la santé et des services sociaux (CRSSS) dans chaque région. Les trois parties représentées étaient les établissements publics, les organismes communautaires et les « corps intermédiaires », c'est-à-dire les établissements privés, les municipalités, les syndicats, les commissions scolaires, la police, etc. Le but de ces comités était de définir les plans d'organisation de services (POS) pour chaque région. Ce qui devait être un exercice de quelques mois a pris dans quelques régions près de deux ans. Tous ou presque reconnaissent l'acquis important que représentent ces comités tripartites (remplaçant les commissions administratives des CRSSS) sur le plan démocratique, confirmant : «que la santé mentale n'est plus exclusivement l'affaire des psychiatres et des institutions psychiatriques, ni non plus du seul réseau étatique " (RRASMQ, $1990: 4$ ). Mais les rencontres ont permis de faire ressortir clairement l'écart des moyens, du fonctionnement et des approches entre les différentes parties.

$D^{\prime}$ ores et déjà, nous sommes en mesure d'affirmer que la rencontre du communautaire et du réseau public - sans être tout à fait du troisième type - $s^{\prime}$ est avérée difficile. Cette rencontre nous force à requestionner nos rapports à l'État. Car pour plusieurs, on n'exagère rien en disant que le choc a été brutal. (RRASMQ, $1990: 5$ ) 
Les points sur lesquels le "choc des cultures "s'est manifesté touchaient notamment les approches épidémiologiques et communautaires, la définition même du "communautaire " si souvent galvaudée et utilisée pour justifier la désinstitutionnalisation et les rapports entre les modèles institutionnel et communautaire (RRASMQ, 1991 : 36-41). Au-delà des points de litige sur le contenu même des PROS il y a eu maints affrontements sur le rythme de travail, sur le mode de représentation dans les comités tripartites et sur l'absence de débats de fond, qui, selon le RRASMQ, évacuait les vrais enjeux de ces débats, c'est-à-dire la nature même des besoins, les déterminants socio-économiques et politiques, le rôle des institutions et la nature des soins à apporter.

En dépit de ce bilan relativement négatif, toutes les parties concernées estiment important de poursuivre ces rencontres, même si l'on ne peut parler de véritable partenariat, dans la mesure où les parties sont inégales et $\mathrm{n}^{\prime}$ ont pas réussi partout à développer un véritable mode de travail respectueux des natures foncièrement différentes. II reste encore beaucoup de chemin à parcourir. C'est sans doute pourquoi le ministre a imposé un moratoire jusqu'au printemps de 1992 pour la réalisation des $P O S$ régionaux.

Il demeure qu'il y a eu, dans certaines régions, des efforts très sincères teintés de respect et d'ouverture d'esprit tant des ressources alternatives que des établissements publics. De plus, il y a des gains de la part des ressources alternatives (RRASMQ, 1991 : 44-45) : la reconnaissance de leur existence; les alliances nouvelles pour remettre en question le modèle institutionnel centralisateur; l'acceptation par les ressources publiques du principe de la complémentarité des ressources publiques et de la communauté, mieux placée pour offrir des services adaptés.

\section{L'exemple du maintien à domicile}

Dans ce champ d'activité les origines des partenaires sont différentes et les visions sont moins divergentes. D'une part les CLSC, centres de jour et centres d'accueil sont plus récents et ont, tout au moins certains d'entre eux, développé une approche communautaire. D'autre part, les organismes communautaires œuvrant dans ce champ s'appuient sur une tradition de bénévolat qui n'a commencé que récemment à affirmer une place autonome et un droit au chapitre. En fait, plusieurs de ces organismes communautaires sont non pas des initiatives autonomes du milieu, mais des créations des établissements publics. Ces derniers (surtout les CLSC) ont souvent mis sur pied des centres de bénévolats, des groupes d'aide aux foyers qui devaient 
compléter leurs ressources limitées pour répondre à une clientèle plus nombreuses et plus lourde. Le financement des CRSSS est aussi déterminant pour la plupart de ces organismes.

$D^{\prime}$ 'une recherche récente* portant sur la collaboration entre organismes communautaires et CLSC œuvrant auprès des personnes âgées à domicile, il se dessine un portrait des tentatives de partenariat, et à la limite certains modèles. II ressort aussi des facteurs déterminants, pour un véritable partenariat. Ces facteurs pourront servir à définir les conséquences pour la formation en travail social.

\section{Les modèles de collaboration}

Considérant les caractéristiques déjà décrites des organismes communautaires et des établissements publics qui se retrouvent toujours à des degrés très variables, on peut identifier deux modèles de collaboration : ce sont en fait deux pôles dans un continuum qui laissent plusieurs variantes entre ceux-ci. Ces deux pôles sont le partenariat réel et équitable d'une part et le " pater-nariat » d'autre part.

Le partenariat réel peut se définir comme un rapport égalitaire et équitable entre deux parties différentes par leur nature, leur mission, leurs activités, leurs ressources et leur mode de fonctionnement. Dans ce rapport les deux parties ont des contributions mutuelles différentes mais jugées mutuellement également essentielles. Le partenariat réel est donc fondé sur un respect et une reconnaissance mutuelle des contributions et des parties impliquées dans un rapport d'interdépendance. L'objet du partenariat devient un échange de services ou de ressources de nature différente mais de poids ou de valeurs comparables ou reconnues comme telles par les parties impliquées. L'objet du partenariat peut aussi être la création commune d'un projet ou d'une ressource (joint venture). Enfin, ce partenariat laisse place à des espaces de négociation (Harnois, 1987) où les parties peuvent définir leur projet commun.

Deux situations peuvent être marquées de cette étiquette de partenariat.

1. Les organismes communautaires existent déjà, ont une mission définie indépendante de la volonté du CLSC et ont une certaine autonomie financière. Ils peuvent avoir des sources indirectes ou directes de financement du CLSC : contrat de services objet de la collaboration, photocopie, espaces sous-loués, accès à des salles de réunion, soutien technique ponctuel d'un intervenant communautaire.

2. Les organismes ont été créés avec l'aide du CLSC, mais ils en ont acquis depuis une certaine autonomie politique et financière, sans pour autant avoir coupé tout lien financier. 
Ces organismes communautaires ont une assez grande autonomie par leur crédibilité, leur enracinement, leur soutien bénévole (et membres), leur permanence et leur financement. Leurs structures de direction sont solides et indépendantes. Ils peuvent recevoir un soutien technique du CLSC parce qu'ils sont perçus comme étant complémentaires au CLSC. Le CLSC a alors une approche communautaire démocratique (à des degrés variables) et perçoit les ressources du milieu comme des atouts et des forces, mais non comme des instruments de sa propre politique. Ces CLSC favorisent une concertation avec le milieu, mais tentent de ne pas garder le contrôle de ces tables pour plutôt en être un acteur « comme les autres » ou en partager le leadership.

Le rôle des intervenants communautaires de CLSC devient celui de soutien technique mais aussi de formateur, dans une orientation conscientisante visant l'autonomie des organismes, en développant leurs responsabilités et leurs pouvoirs. L'intervenant définit son mandat clairement avec les organismes et avec le CLSC en prévoyant des périodes précises et des évaluations périodiques, afin de ne pas devenir un soutien permanent. L'intervenant évite surtout de devenir l'intermédiaire officiel entre le CLSC et l'organisme communautaire, voire le négociateur des deux organismes.

Le « pater-nariat » est le pôle opposé du continuum marqué par un rapport inégalitaire entre parties différentes et inégales. Dans ce modèle, les parties peuvent avoir des contributions mutuelles différentes et parfois même jugées essentielles, mais le plus souvent dans un but dominant. La partie dominante est l'établissement du réseau public qui considère comme importante, voire essentielle, la contribution de l'organisme communautaire, dans la mesure où elle s'insère en complémentarité de sa propre mission. Donc, il n'y a plus indépendance égalitaire, mais plutôt complémentarité à sens unique. La contrepartie, dans l'échange de ressources ou de services, n'est pas équitable. II s'agit donc d'un rapport utilitaire défini avant tout par les exigences de l'une des parties impliquées, qui "sous-traite " ou déverse une partie de ses responsabilités à un organisme communautaire.

C'est évidemment le pôle le plus fort qui marque le plus de pratiques. Dans ces situations, les CLSC créent des organismes bénévoles pour compléter leur travail devant le débordement d'une clientèle plus lourde et plus nombreuse.

Ces groupes ont généralement une forte dépendance à l'égard des CLSC. Lorsqu'ils affirment une certaine volonté d'autonomie, on (les CLSC) dit qu'ils traversent une "crise d'adolescence ». Ils existent dans des quartiers où il y avait de faibles ressources pour les personnes âgées. Les CLSC qui les créent ont habituellement une approche communautaire plus technocratique que démocratique, c'est-à-dire qui 
vise l'utilisation des ressources du milieu sous la direction professionnelle de l'établissement public. Ils ont une politique d'intégration des ressources du milieu à leurs propres objectifs. Ces CLSC favorisent aussi une concertation plus grande entre les organismes et avec les CLSC. Ils créent, soutiennent, maintiennent et dirigent ces tables de concertation. Enfin, les CLSC ont peu de connaissance et donc peu de tolérance à l'égard des critiques et des revendications des organismes communautaires. Ces relations sont souvent caractérisées par un dialogue de sourds.

Le rôle des intervenants communautaires dans cette dynamique se caractérise par trois dimensions principales :

- l'intervenant est instigateur, soutien technique et souvent " porteur à bout de bras " des groupes;

- l'intervenant occupe souvent des fonctions officielles au conseil d'administration de l'organisme. On maintient ainsi le lien structurel entre l'organisme et le CLSC;

- l'intervenant a généralement une orientation qui favorise l'atteinte de l'objectif de production, le développement de services et la bonne gestion afin de répondre aux besoins du milieu définis par le CLSC.

Donc, dans un pôle on trouve une autonomie et une marge de manœuvre des organismes communautaires qui sont limitées et qui favorisent le débordement du public sur le communautaire. Dans l'autre pôle on trouve un organisme plus autonome qui peut se positionner comme une véritable solution de remplacement à l'institution publique; cela favorise une collaboration égalitaire $(\mathrm{H}$. Tremblay, 1987 : 28).

On comprend les visions qui peuvent s'opposer. Le secteur communautaire souhaite demeurer autonome, extérieur au réseau public et non récupéré. Le secteur public voit le secteur communautaire comme un apport (en échange d'une légitimité accrue par le financement public), une partie intégrante au réseau, avec une autonomie réduite mais une stabilité plus grande (COCQ, 1988; Robert, 1989 : 47). Cette vision du secteur publique suscite la critique du secteur communautaire qui se dit reconnu uniquement pour les services offerts mais non pour son existence propre, son mode de fonctionnement et ses valeurs différents.

\section{Les facteurs déterminants}

Évidemment, les statuts et le financement sont parmi les nombreux éléments qui peuvent rendre un partenariat difficile, mais les 
recherches (Godbout, Leduc, Collin, 1988) ont montré malgré tout que c'est possible. Un certain nombre de conditions doivent exister.

On peut distinguer des facteurs institutionnels et des facteurs humains qui influencent les résultats de la collaboration. La réussite de la collaboration se mesure à partir d'abord de l'impact sur les personnes âgées (quantité et qualité de l'aide), puis de l'impact sur les organismes collaborateurs (dynamisme interne, amélioration du climat de travail, augmentation de la productivité, de la crédibilité, de l'enracinement, de l'accessibilité des services, etc.).

\section{Les facteurs institutionnels}

- Au départ, il doit y avoir une évaluation de besoins réels et non des préoccupations strictement institutionnelles. La collaboration vise à promouvoir et à soutenir le maintien à domicile des personnes âgées dans le respect de leur dignité et dans des conditions de vie de qualité. La collaboration doit évidemment éviter la duplication de services. Or, on constate souvent que la planification ou les programmes cadres dictent avant tout la volonté des établissements publics d'établir des collaborations avec des organismes bénévoles. Les organismes manifestent souvent un souci sincère pour la clientèle, mais la motivation première de tenter une collaboration avec un partenaire relève souvent du souci de leur développement organisationnel. Ce souci est dicté soit par ses membres ou sa direction, soit extérieurement par les contraintes et conditions des bailleurs de fonds (I'État provincial, le CRSS, Centraide, etc.).

- L'enracinement et la force des organismes en présence sont importants pour assurer une collaboration équitable et une participation active du milieu. Cette intégration au milieu crée une pression positive du milieu et rend I'organisme (communautaire ou public) plus transparent et plus imputable. Le faible enracinement du CLSC peut contribuer à un manque d'ouverture sur le milieu et à une mécompréhension des organismes communautaires, de leur culture, de leurs limites, de leurs objectifs et de leurs attentes. C'est cette méconnaissance qui est souvent reprochée par les organismes communautaires. Par ailleurs, les expériences de partenariat réel sont fondées justement sur un bon enracinement des parties en présence et une bonne connaissance mutuelle.

- Les attentes et les objectifs de collaboration doivent être clairs pour chacun des organismes dès le départ. Les organismes qui n'ont pas fait leur propre effort d'autodéfinition sont souvent une proie facile pour le vis-à-vis qui sait clairement ce qu'il veut en tirer. Les organismes communautaires observés n'ont pas toujours développé 
cette clarification surtout s'ils sont récents et s'ils doivent leur création au CLSC. Leurs objectifs sont alors définis par rapport à ceux du CLSC qui les a créés.

- La négociation qui se fait autour de mandaís clairs soutenus par les instances officielles des organismes a plus de chance de parvenir à une entente équitable. Cela assure aussi la transparence et la participation des instances élues dans le processus, ajoutant ainsi plus de poids à l'entente.

- L'entente ou le protocole entre les organismes gagne à être écrit. On y précise les objectifs, les responsabilités mutuelles et les modalités d'application (critères d'admissibilité, financement, durée, etc.). Les chances sont alors plus grandes d'éviter les erreurs d'interprétation. Habituellement, l'écriture d'une entente amène à y prévoir une évaluation périodique et des moments d'échange. Cette communication régulière est fondamentale pour éviter la détérioration de malentendus ou de situations conflictuelles. Ces relations ouvertes et transparentes permettent de faire le point et d'ajuster les modalités de l'entente. L'inverse peut amener un climat malsain qui brûle des énergies inutilement.

- La reconnaissance mutuelle des rôles et des limites des organismes est essentielle. Dans un esprit d'interdépendance ils doivent être à l'écoute des besoins et des capacités de l'un et l'autre. C'est cette reconnaissance qui est encore si difficile à acquérir de part et d'autre. La connaissance est à la base de cette reconnaissance.

- Le respect de l'autonomie des organismes communautaires par les organismes publics est fondamental, surtout lorsque l'organisme communautaire est une création du CLSC. Cette situation de paternité n'est pas facile à assumer, surtout au moment où l'organisme communautaire prend une certaine autonomie. Il peut y avoir à ce moment des tensions qui ne se résolvent pas toujours, même avec le temps.

- Une table de concertation des organismes du milieu peut aider à la collaboration entre deux organismes pour maintenir un certain équilibre. Les tables de concertation et regroupements sectoriels permettent de créer un rapport de force qui soutient habituellement les organismes communautaires dans leur collaboration avec les établissements publics. Cela crée ainsi des rapports plus égalitaires.

Le droit de paternité sur l'organisme communautaire et les besoins de soutien professionnel ou technique de celui-ci créent au départ une relation inégale. Les politiques gouvernementales et les contraintes budgétaires créent aussi des pressions déterminantes sur le CLSC et donc sur les relations qu'il peut entretenir avec les organismes communautaires. Pour le CLSC, ces organismes sont de plus en plus impor- 
tants dans la réalisation de ses objectifs. L'approche communautaire prônée par la Fédération des CLSC ajoute une référence professionnelle et idéologique pour rationaliser l'utilisation des ressources communautaires. Le CLSC impose donc ses intérêts et réduit les organismes communautaires à une valeur instrumentale pour parachever la réalisation du programme du CLSC. Les revendications d'autonomie des organismes communautaires ont peu de poids si elles ne sont pas soutenues par des regroupements provinciaux ou des tables de concertation locale. La tradition des organismes communautaires offrant des services pour soutenir le maintien à domicile des personnes âgées $n^{\prime}$ est pas militante; elle vient plutôt d'une tradition caritative et de bénévolat traditionnel. Les organismes bénévoles sont plus facilement isolés dans leur relation de collaboration avec les CLSC et sont donc dans une position de faiblesse, d'autant plus qu'ils doivent souvent leur existence et leur survie aux CLSC avec lesquels ils veulent négocier d'égal à égal. L'enjeu pour le CLSC est donc de pouvoir respecter sa mission et offrir des services, notamment à la population âgée à domicile, dans les limites des contraintes politiques et budgétaires. Le respect de l'autonomie et de la nature spécifique des organismes communautaires ne devient alors qu'un facteur utilitaire au service de la mission de l'établissement public.

L'enjeu pour les organismes communautaires, c'est évidemment le respect de leur intégrité et le développement de leurs activités en respectant la volonté de leurs structures démocratiques. Les organismes communautaires tirent souvent avantage de la collaboration avec les établissements publics (personnes-ressources, soutien technique) qui contribuent à leur développement et à leur crédibilité aux yeux de la clientèle. Mais cette crédibilité, liée à la qualité des services offerts, a un prix : le respect de la mission de socialisation et de prévention. L'enjeu est donc lié à la capacité des organismes communautaires de se développer de façon autonome et de garder une approche, un mode de fonctionnement et des ressources humaines qui en font une autre solution possible de qualité pour la clientèle. C'est là la complémentarité réelle avec les CLSC.

\section{Les facteurs humains}

- La qualité, la clairvoyance, la franchise et l'enracinement des personnes en cause peuvent contribuer au succès de la négociation. Le respect mutuel et les bons rapports personnels sont toujours essentiels mais insuffisants pour assurer la réussite.

- Les intervenants communautaires sont aussi des facteurs de réussite dans leur rôle auprès de leur CLSC et auprès de l'organisme 
communautaire. Dans le CLSC, les intervenants communautaires peuvent jouer un rôle important pour sensibiliser le personnel à la réalité des organismes communautaires (leur existence, leur force et leurs limites, leur façon de travailler). Les intervenants peuvent aussi avoir une influence importante sur le développement des politiques du MAD en général et de collaboration avec les organismes communautaires.

- Dans l'organisme communautaire, la qualité du soutien de l'intervenant (personne-ressource) est importante dans la façon de développer la démocratie et la prise de distance par rapport au CLSC, surtout si celui-ci a été à l'origine de l'organisme. L'intervenant doit avoir un mandat très clair, circonscrit dans le temps pour agir comme source d'information et de formation auprès du groupe (s'il y a lieu) sans devenir négociateur (pour aucune des deux parties). Cela incite les instances respectives à se rencontrer sans intermédiaire.

- L'intervenant doit éviter de porter à bout de bras l'organisme communautaire afin de le maintenir artificiellement.

Les intervenants communautaires, puisque ce sont ceux qui sont souvent déterminants comme personnes-ressources, doivent travailler à l'intérieur des mandats émanant de la direction des CLSC. Or, ces mandats sont de plus en plus circonscrits dans une optique de productivisme, c'est-à-dire que l'on attend des services précis et des résultats de la part des organismes communautaires. Les intervenants communautaires sont poussés à mettre sur pied ou à soutenir des organismes pour assurer l'atteinte des objectifs des CLSC. Le rôle des intervenants communautaires à l'égard des organismes est un rôle de personne-ressource pour définir et développer la mission, les objectifs, les moyens et le mode de fonctionnement en aidant aussi au financement.

Mais ils dépassent parfois ces rôles. Lorsque les intervenants deviennent des élus de l'organisme et des représentants de l'organisme notamment face au CLSC, il peut y avoir des conflits d'intérêts et donc des difficultés qui contribuent à la confusion. L'intervenant qui dépasse le rôle de personne-ressource n'aide pas l'organisme à long terme et même pas à court terme, malgré l'apparence de facilité (faire soi-même au lieu de développer les habiletés chez les membres de I'organisme). Donc, là où les intervenants ont une trop grande emprise sur les organismes, il risque d'y avoir une relation de dépendance et donc de " pater-nariat ».

L'intervenant communautaire est dans une position difficile entre les besoins de l'organisme de se développer pour mieux servir la clientèle à sa façon et les besoins du CLSC qui attend des services 
précis de cet organisme sans se soucier de son développement à long terme.

Sans nier leurs compétences et leur conscience professionnelles, on peut dire que les intervenants communautaires ont aussi besoin de compétences pour négocier leur rôle avec leur employeur objectif (le CLSC) et leur mandataire effectif (I'organisme communautaire). Cela peut être accompagné du souci de toujours mieux connaître le milieu et les organismes en partageant cette connaissance dans le CLSC afin de sensibiliser les autres intervenants et la direction.

Les intervenants communautaires et les autres personnes en cause ne voient pas toujours comment ils font naître et imposent gentiment leur culture organisationnelle et leurs objectifs. Animés par une bonne volonté, les intervenants ne voient pas toujours le cul-de-sac à long terme de leur intervention lorsqu'ils poussent les organismes à développer certains services jadis dévolus aux CLSC. Le manque de confiance, la méconnaissance et l'insécurité poussent autant les intervenants des CLSC que les intervenants des organismes communautaires dans une relation de dépendance qui se rabat sur les exigences du réseau public.

\section{Les déterminants de la formation en travail social}

Jamais la capacité de changement n'aura été autant sollicitée auparavant dans I'histoire de la profession du travail social. Les réformes, certes, se sont succédé depuis plus de trente ans, mais nous sommes entrés dans une période d'incertitude, fortement ressentie par les intervenants et intervenantes, qui ne va pas s'atténuer. Les données évoluent, changent et augmentent constamment. Certaines données étaient présentes déjà, mais n'apparaissaient pas avec autant d'acuité (les clientèles d'origines ethniques variées, les conséquences socio-sanitaires de la pollution, les pressions budgétaires, les liens entre les services publics et le milieu, les conséquences de la désinstitutionnalisation tant des ex-psychiatrisés que des personnes âgées et handicapées, la violence conjugale, I'itinérance), alors que d'autres sont nouvelles (les conséquences du SIDA).

Devant ces changements, et plus particulièrement cette tendance à la concertation intersectorielle et au partenariat entre secteurs public et communautaire, il y a un certain nombre de changements à apporter dans la formation en travail social. Au-delà des enseignements de méthodes et de techniques spécialisées qui se développent déjà (intervention auprès des victimes d'agressions sexuelles ou autres, intervention auprès des personnes atteintes du SIDA), il y a des changements $\mathrm{d}^{\prime}$ attitudes humaines et organisationnelles qu'il faudra susciter... ou 
retrouver. Car comme souvent dans des périodes de changement rapide, on peut revenir à des valeurs sûres et à des principes qui demeurent pertinents.

\section{Les changements organisationnels}

Dans presque toutes les écoles de service social nord-américaines, l'intervention communautaire a été un peu "tassée " depuis les années 70. Les modèles conflictuels ont été remplacés par les approches plus douces et individualisantes dans les années 80 , alors que le secteur communautaire se développait de façon presque géométrique. Le colloque d'octobre 1986 à Victoriaville ( Fais-moi signe de changement ") a été une occasion de constater ce vent d'énergie nouvelle... et de maturité. Les acquis plus solides mais toujours fragiles du mouvement communautaire de plus en plus diversifié en font maintenant une réalité que l'on ne peut pas ignorer dans la formation. Et c'est justement la diversité grandissante qui en fait un acteur qui doit prendre une place prépondérante dans la formation, comme dans le développement socio-sanitaire et économique (car l'économique a aussi repris une place plus importante dans les années 80 de l'économisme néo-libéral).

Cela demande aux écoles de développer des liens plus nombreux avec les organismes communautaires à des fins de stage, de contribution à l'enseignement (expertise reconnue), de recherche et de concertation vers des changements socio-politiques. Pour développer ces rapports, les écoles devront faire montre d'ouverture, de souplesse (dans leurs critères d'excellence) et de profond respect à l'égard de connaissances et de pratiques différentes de celles du réseau public. En fait, les écoles de service social peuvent même contribuer à la reconnaissance et la crédibilité face à l'État, tant recherchées par les organismes communautaires.

\section{Les changements individuels}

Au chapitre de la formation des futurs intervenants et intervenantes, le développement des savoir, savoir-être et savoir-faire devra renouveler son insistance sur certaines connaissances, attitudes et habiletés. À ces trois savoirs traditionnels on pourrait ajouter le savoirdire pour prendre position plus souvent sur les enjeux socio-politiques avec les organismes communautaires. On pourrait aussi revaloriser une qualité fondamentale mais trop souvent oubliée dans l'intervention : le savoir-écouter. L'insistance des écoles et des corporations professionnelles à affirmer leur expertise et leur identité propre (sur- 
tout en travail social) a eu tendance à être corporatiste. Certes, dans toute relation égalitaire les parties doivent avoir une perception claire de ce qu'elles sont elles-mêmes pour entrer en contact avec l'autre. Cet équilibre entre cette auto-définition et la capacité d'écoute, d'ouverture et de négociation est difficile à atteindre, mais aussi essentiel dans une relation de partenariat réel. Toutes les recherches le démontrent. Donc, le développement d'une identité forte mais non dominante est un élément de base dans la formation.

Sur le plan des connaissances (savoir), la formation pourrait se développer dans les analyses statiques (portrait) et dynamiques (les rapports sociaux) des milieux. La connaissance des ressources communautaires, leurs origines, leur mission, leurs objectifs, leurs activités et leur culture organisationnelle est fondamentale. L'environnement des politiques sociales est aussi une dimension importante de ces connaissances.

Sur le plan des attitudes (savoir-être), la culture de l'écoute empathique et du respect de l'autre sera toujours à la base de l'intervention. En corollaire, la souplesse, la tolérance, la patience (face à des rythmes différents et des cultures organisationnelles différentes), la disponibilité, la transparence et la franchise sont toutes des attitudes importantes à maintenir. On pourrait ajouter que I'humilité, mais non la fausse modestie, est une attitude qui doit côtoyer le professionnalisme au sens noble du terme, c'est-à-dire la compétence. Mais le corporatisme protectionniste $n^{\prime}$ a pas sa place dans une relation de partenariat qui exige une ouverture d'esprit essentielle à la créativité.

Sur le plan des habiletés (savoir-faire) le travail d'équipe multisectoriel et multiculturel serait essentiel pour développer des projets communs avec des parties d'origine et de nature différentes.

Les habiletés à négocier les conflits sont aussi très importantes dans une ère de partenariat. Les espaces de négociation ne sont pas toujours très grands et les positions de départ peuvent être très lointaines. Or, le conflit n'est pas nécessairement négatif en soi. Il peut être envisagé comme une occasion favorisant la prise de conscience et la créativité pour trouver de nouvelles solutions. Dans toutes les expériences positives de partenariat, il y a eu des attitudes d'ouverture et de la persévérance pour aller au-delà des positions de départ. Mais souvent les parties en conflit ont dû vaincre des préjugés profonds. Dans ce sens l'art de la négociation se pratique, s'acquiert et peut amener des retombées positives pour les parties. 


\section{Conclusion}

Le partenariat est un défi de taille entre des parties si différentes. Les discours généreux peuvent ne pas couvrir des intentions sincères, mais plutôt des calculs politiques. II ne faut pas nier les intérêts divergents qui peuvent s'affronter dans la pratique. Les expériences malheureuses et heureuses ont démontré que la pratique du partenariat est difficile à atteindre... mais sans doute inévitable. Le défi est d'en faire une relation équitable plus qu'égalitaire afin d'inventer des solutions nouvelles pour affronter des problématiques nouvelles et des conditions de plus en plus difficiles. Cela prend du temps et exige un apprivoisement patient.

Par ailleurs, certaines études (Conseil des affaires sociales, 1989) nous mettent en garde : il ne faut pas présumer que toutes les communautés ont les mêmes capacités à développer les ressources qui peuvent être des partenaires vis-à-vis de l'État. Le niveau de développement de certaines régions et de certains quartiers est extrêmement faible et ne peut assurer les ressources communautaires qui pourraient être complémentaires. Ce sont justement les milieux les moins développés en ressources qui ont les populations avec les plus grands besoins. Le partenariat, selon lequel, comme le réclame le RRASMQ (1991), les services publics doivent être considérés comme complémentaires au milieu, pourrait être une panacée pour justifier un laisserfaire et un déséquilibre encore plus grand.

La formation en travail social sera de plus en plus déterminée par ce contexte. On ne peut plus former massivement des intervenantes pour travailler en protection de l'enfance. Le syndrome de la DPJ que certaines étudiantes dénoncent doit faire place à la polyvalence, à la multifonctionnalité et à la démonopolisation du secteur public. Sans penser à la « communautarisation » de l'État (Robert, 1989), qui peut galvauder un peu trop le communautaire, on peut espérer une rencontre constructive de deux univers condamnés à se côtoyer pour répondre à des besoins grandissants.

\section{Note}

\footnotetext{
* Les conclusions dans ce domaine sont issues d'une recherche sur les collaborations entre organismes communautaires et publics œuvrant auprès des personnes âgées à domicile. L'équipe de recherche, dirigée par Jean PanetRaymond, est formée de Denis Bourque, Sylvie Cameron, Bernard Ngamo et Aline Vandal. Le rapport final a paru en novembre 1991 sous le titre Partenariat ou "pater-nariat "?, Jean Panet-Raymond et Denis Bourque, Université de Montréal.
} 


\section{Références bibliographiques}

Bailey, M., R. Seyd et A. Tennant (1989). Local Health and Welfare. Is Partnership Possible? United Kingdom : Gower Aldershot.

BOURQUE, D. (1988). Des alliances... mais à quel prix ? Conférence prononcée au colloque organisé par le CRSSSMM, 28 septembre.

BouRQue, D. (1991). "Le partenariat! Quel partenariat !", Inter-action communautaire, janvier : 8 et 9.

Coalition des organismes communautaires du Québec (1988). Pour la reconnaissance de l'action communautaire.

Conseil des affaires sociales (1989). Deux Québec dans un. Rapport sur le développement social et démographique, Québec.

Corporation de développement communautaire des Bois-Francs (1986). Faismoi signe de changement. Actes du colloque provincial sur le développement communautaire, Victoriaville, 16-18 octobre.

CôtÉ, M.-Y. (1990). Une réforme axée sur le citoyen. Québec.

CRSSSMM (1988). Des alliances... mais avec qui ? Rapport du colloque du 28 septembre.

Godbout, J., M. Leduc et J.-P. Coluın (1988). La face cachée du système. Annexe $n^{\circ} 22$, Commission d'enquête sur les services de santé et les services sociaux, Québec.

Godbout, J. et J. Guay (1989). Le communautaire public. Montréal, INRS.

Harnols, G. (1987). Pour un partenariat élargi. Rapport du comité d'étude sur la santé mentale, Québec.

LesemanN, F. et J. Lamoureux (1988). Les filières de l'action sociale. Annexe $n^{\circ} 24$, Commission d'enquête sur les services de santé et les services sociaux, Québec.

Panet-Raymond, J. et A. Vandal (1990). Le maintien à domicile à l'heure du partenariat entre organismes communautaires et CLSC. Conférence prononcée au congrès du Regroupement des intervenantes et intervenants en action communautaire en CLSC, 25 mai.

ParazelLi, M. (1988). " L'action communautaire autonome en tutelle ! ", Interaction, décembre : 1 et 2 .

Regroupement des ressources alternatives en santé mentale du Québec (1990). Le choc... Document d'introduction au bilan de l'an 1 de la politique de santé mentale, mai 1990.

Regroupement des ressources alternatives en santé mentale du Québec (1991). Le choc des cultures. Bilan synthèse de l'expérience de participation aux comités tripartites, juin.

ROBERT, L. (1989). "Le partenariat entre le réseau institutionnel et la communauté : un paradigme à définir ", Nouvelles pratiques sociales, PUQ, vol. 2, $\mathrm{n}^{\circ} 1: 37-52$.

SAINT-ONGE, J. (1990). "L'État-providence agonise... vive le communautaire et la privatisation », Le gérontophile, vol. 12, n 4 : 16-19.

Shragge, E. et T. Ladouceur (1988). Community Initiate Health and Social Services. Annexe $\mathrm{n}^{\circ} 7$, Commission d'enquête sur les services de santé et les services sociaux, Québec. 
TREMBLAY, F. (1987). Désengagement de l'État et transfert des interventions aux groupes communautaires. Conférence prononcée au $8^{\mathrm{e}}$ colloque de l'AQG (Association québécoise de gérontologie).

Tremblay, H. (1987). Les ressources communautaires. Problématique et enjeux. MSSS, Québec.

TREMBLAY, J. (1987). État de la situation présentée au service de soutien aux organismes communautaires concernant les organismes subventionnés en 1986-1987. MSSS, Québec.

VAillancourt, Y., D. BourQUe, F. David et E. Ouellet (1988). La privatisation des services sociaux. Annexe $n^{\circ} 37$, Commission d'enquête sur les services de santé et les services sociaux, Québec. 